\title{
Health Diagnosis Using Wban Technology
}

\author{
G. Swaapnika ${ }^{1}$, Dr. N. Chandra Sekhar Reddy ${ }^{2}$, SVS. Hanumanth Rao ${ }^{3}$, \\ I.Surya Prabha ${ }^{4}$ \\ ${ }^{I}$ M.Tech Student, IT Dept., Institute of Aeronautical Engineering, HYD-500043, AP, India. \\ ${ }^{2}$ Professor, CSE Dept., Institute of Aeronautical Engineering, HYD-500043, AP, India. \\ ${ }^{3}$ Assoc., Professor, CSE Dept.,Institute of Aeronautical Engineering, HYD-500043, AP, India. \\ ${ }^{4}$ Assoc., Professor, CSE Dept., Institute of Aeronautical Engineering, HYD-500043, AP, India.
}

\begin{abstract}
Invention of the low power consuming wireless sensors are raising the emergence for the usage of wireless body area networks (WBAN).WBAN is widely used in many applications such as entertainment, gaming, army etc this is widely used for active applications as it provides instant monitoring .So, this is very useful for healthcare sensing which provides effective monitoring with the help of tiny sensors. In, this paper we discuss about the architecture of health monitoring system using WBAN, how to overcome the path attenuation, devices used in this WBAN, body sensor network and security.
\end{abstract}

Keywords: WBAN, Health monitoring system (HMS), Path Attenuation, Body sensor network, security.

\section{Introduction}

Now a days health is the at most concern for the people in our country due to the increasing population the health problems are also getting worse due to their change of lifestyle and they are not even having time to visit a doctor and sometimes during the times of emergency the doctors may not be available so keeping this in mind our technology has new advancements in the field of health so as to save people from problematic situation. This technology is helping the old who are weak from going to the clinics regularly they can happily verify their health condition by sitting in their home. This WBAN uses tiny sensors for determining the health condition which are placed on or around the body. The sensors are placed on the body or around it and these sensors collect the data regarding the health and then after this the WBAN uses data transfer mechanisms like bluetooth, wi-fi, zigbee for giving the data to the doctor. this also uses several devices such as sensor shirt, watch sensor, abdominal belt etc to collect the important data from the patients body and transfer it to the medical practitioner. The path loss depends on the distance of the transmitter and receiver. The different types of sensors used for different body parts and based on body communication we use different antennas. The sensors whish are placed on the body collect the data about our body now to transfer the data to the physician first it should send the data to the remote server using a base station transceiver(BST) this is then connected to the sever pc. The health monitoring system is component based so it consists of bluetooth and smart phone for maintaining patients health condition and his location with help of a accelerometer which acts as intelligent control node(ICN).As we have different types of architectures for WBAN such as sensor shirt, watch sensor, abdominal belt etc we gather data from sensors and using zigbee which is more power efficient than bluetooth that data will be transferred to doctor using a BST which is connected to the server pc. The sensor shirt is a multi-hop sensor network.

\section{Solution For The Improvement Of The Current Medical Monitoring Scenario}

In the current system the doctor will not have continuous observation of the patient and he can't monitor the patient when he is off to remote places so in case of any emergency the doctor will not be available so in order to prevent this drawback we should switch to the health monitoring system(HMS) in which the doctor and patient can communicate using remote sensing technology.

Different communication standards used by WBAN are as follows:

Zigbee- Zigbee is a low power consuming communication standard than compared to bluetooth and it is almost collision free as it uses avoidance schemes to prevent collisions. Security is provided by the encryption techniques of zigbee controllers.

Bluetooth-The data rate in Bluetooth is $3 \mathrm{Mbps}$ and range of $10 \mathrm{~m}$. It has high bandwidth and low length; due to this reason Bluetooth standard is used in the HMS monitoring. The disadvantage in this Bluetooth is high power consumptions. For this reason this is not widely used in HMS.

Medical Implant Communications Service (MICS)- This is the most widely used standard in WBAN communication because it is highly efficient than the bluetooth and consumes less power. It collects signal from different sensors on-body and give it to the BST. 


\section{Architecture Of HMS}

The HMS architecture mainly consists of two main parts one is the personal server(PS) and the other is the medical server(MS).This medical server is the most complex part of the architecture and it provides service to thousands of the users. Now, in this firstly the user wears a set of sensor nodes on the body and this sensors collect the relevant data and send the data to the PS by using the above mentioned communication standards. The PS is implemented on a PDA it gives audio or graphical data to the users now the data is sent to the MS from the PS using telephone network or cell phones etc. The data from the MS is sent to the physician and he/she can access the data from any place using the internet. In case of any emergencies a server agent will inspect the uploaded data and produce an alert so that necessary actions can be taken place in order to save the life of the user. In case of a normal check up after the physician examines the data provided he give back the necessary advice or prescription to the patient through the internet which will be easily given to the user by saving a lot of time to both the physician as well as the patient.

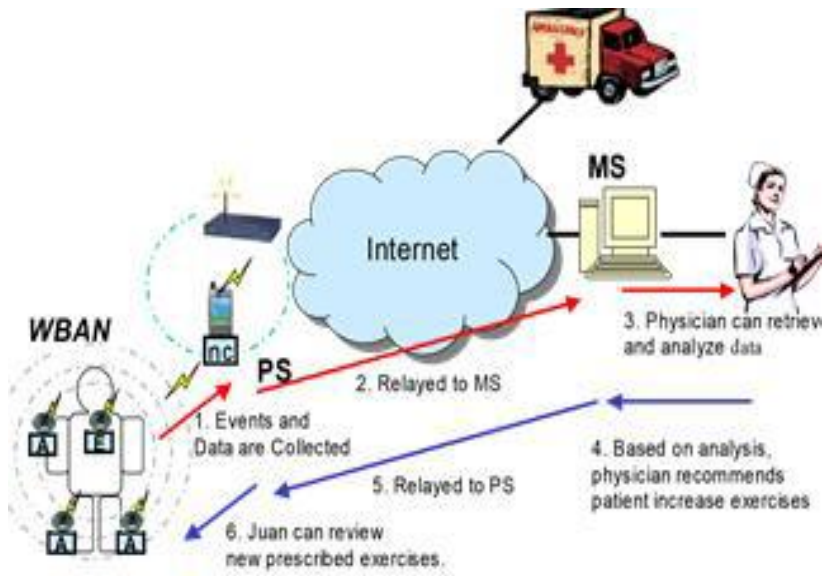

If we come into the detail of how the data is given to the PS in which form it is as follows .Firstly the network coordinator(NC) acts as interface between the person and the PS helps in using the data communication standards sensor nodes get initial commands and give response to the queries from PS. The nodes collect raw data continuously and send processed data to the PS.

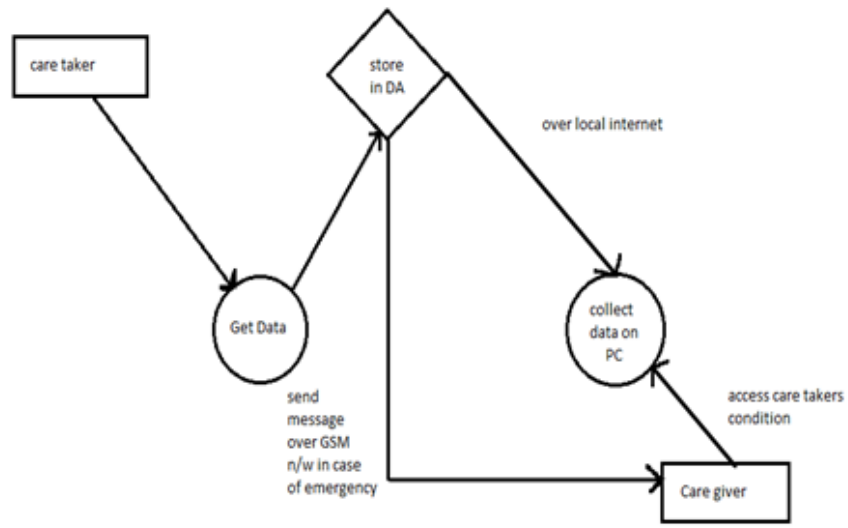

Data Flow Diagram Of The System

As shown in the above data flow diagram the events that mainly occur in this architecture are sampling, processing, storing and communicating. In case of emergencies the GSM is used in order to alert the doctors or the nurse in the clinic by using an alarm. The sensor nodes and network coordinator software is implemented in the tiny OS environment.

\section{Different Types Of Sensors Used}

In this architecture many types of body sensors are used to get relevant data the different types of sensors used are wearable watch, wearable shirt, oxi-meter, chest belt, abdominal belt etc. These sensors are used to get important signs such as heart beat rate, glucose level, detection of cancer cells etc also measurement of surrounding temperature, humidity etc. 
The architecture supports 3 types of nodes those are internal nodes, surface nodes and external nodes. By using these nodes sometimes there may be path loss due to few frequency and distance factors.

Internal node - This is fixed inside the body either under the skin or more deeper, Surface node - This node is placed on the surface of the body maximum $2 \mathrm{~cm}$ away, External node - This node is kept few cm's away from the body.

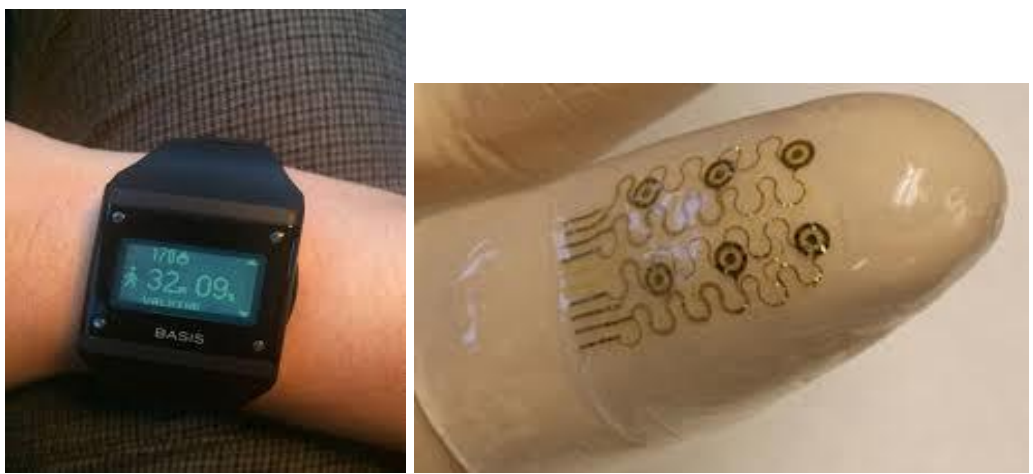

Some wearable sensors

There are 2 types of antennas used by the sensors to get the signals one is electrical and the other is the magnetic antenna. The first one is placed outside the body as it is harmful for the tissues and the second one is placed inside the body as it is not harmful for human body.

\section{Path Attenuation in WBAN}

As we discussed earlier the path loss occurs due to different scenarios and it mostly depends on the frequency and distance. There are two types of communication in-body communication and on-body communication. In in-body communication the probability of getting a normal graph is very less so we can use a $3 \mathrm{~d}$ graph as shown below

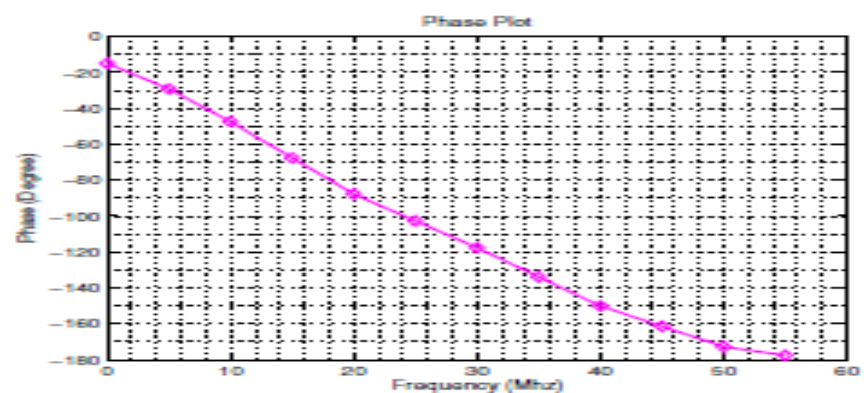

Phase distortion

Now, in the on body communication we can easily draw a graph and in this the path loss is mainly due to the distance and as the distance increases the path loss also increases. In this as the frequency increases the amplitude increases as the channel will have disturbances which results in downfall of signal intensity. In this multiple frequencies occur at multiple phase distortions if the relation is non-linear.

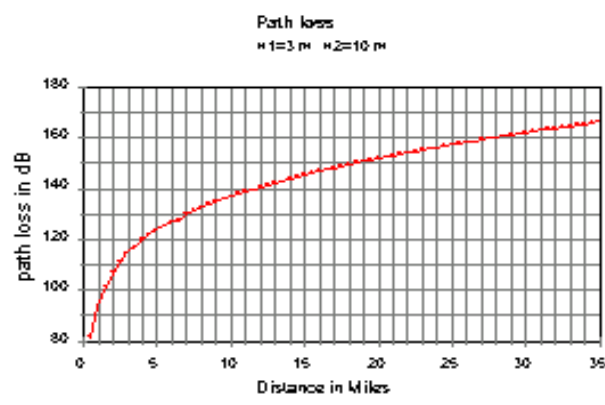

Path loss vs. Distance 


\section{Body Sensor Network}

There are 2 types of body sensor network(BSN) one is the managed body sensor network(MBSN) and the other is the autonomous body sensor network(ABSN).the first one that is the MBSN collects the relevant data from the user and then it gives the information to the third party and that person will take the proper needed decision based on the data collected so as to treat the patient. so the MBSN doesn't have any self intelligence. Now coming to the ABSN this one collects the relevant data and this doesn't depend on the third party to take the decision instead it uses the help of the actuators in order to generate changes on the user's body automatically so it uses the self intelligence technique with the help of actuators. The human++ is a flexible platform which supports the ABSN.

\section{Security Requirements And Solution}

The most important security requirements that should be dealt with while using WBAN are as follows: Authentication- This is to avoid the node impersonation and prevent network compromise in WBAN.

Data Integrity- This is to avoid replay attacks and data modification while the data is moving from one node to the other.

Confidentiality- This is to ensure that the data exchange between the nodes is secretly done or not. Availability- This is to ensure that the data is always available or not as this deals with a highly sensitive and life saving information.

Privacy- As this deals with patients most personal information that is the medical data this should not be disclosed to any un-authorized user. There should be strict rules to achieve this privacy.

Now coming to the solution to achieve this security we have tinysec this implements the link layer encryption to achieve the authentication this is a software based security architecture. This is one of the component of tinyOS. In this the encryption of the data packet including the header is done with a group key common to all sensor nodes and generates a message authentication code(MAC) including the header and is distributed to all the nodes in the network. Hence in this way it provides security in WBAN etc.

\section{Conclusion}

WBAN is the most important technology in today's world for supporting the health monitoring system by using wireless communication with the help of sensor nodes. These sensors are the most important part of WBAN which collect the patients health data and give it to the doctor through different communication standards. The HMS is to the benefit of people they need not go to the doctor all the way for their health check up. This is a very efficient one in the present busy world. This paper consists of the architecture, the different types of sensors, ways to prevent path loss, different types of body sensor networks(BSN), and finally the security issues and a solution for it are discussed.

\section{References}

[1] Joonyoung Jung, et al.,"Wireless Body Area Network in a Ubiquitous Healthcare System for Physiological Signal Monitoring and Health consulting”, International Journal of Signal Processing, Image Processing and Pattern Recognition, 2008.

[2] Emil Jovanov, et al., "Body Area Networks for Ubiuitous Healthcare Applications: Opportunities and Challenges", J Med Syst(2011) 35:1245- 1254, 2011.

[3] P. S. Hall, et al., "Antennas and Propagation for On-Body Communication Systems", IEEE Magazine on Antennas and Propagation. vol. 49 (3), pp. 41-58, 2007

[4] Ulla, S., et al., "A Review of Wireless Body Area Networks (WBANs) for Medical Applications", International Journal of Communications, Network and System Sciences (IJCNS), Vol. 2,No. 8, pp797-803. 2009

[5] Wan-Young Chung, et al., "A Wireless Sensor Network Compatible Wearable U-healthcare Monitoring System Using Integrated ECG, Accelerometer and SpO2”, 30th Annual International IEEE EMBS Conference vancouver, British Columbia, Canada, August 20-24, 2008

[6] Young-Dong Lee, et al., "Wireless Sensor Network Based Wearable Smart Shirt for Ubiquitous Health and Activity Monitoring", International Meeting of Chemical Sensors 2008 (IMCS-12), July 13-16, 2008, OH, USA

[7] Kamya Y. Yazdandoost and Ryuji Kohno, et al., "The Effect of Human Body on UWB BAN Antennas", IEEE802.15-07-0546-00Oban.

[8] Twist04] Twist, "When technology gets personal," BBC News on Science and Technology 2004/12/06

[9] Schmidt02] Schmidt et al., "Body Area Network BAN--a key infrastructure element for patient-centered medical applications," Biomedizinische Technik. Biomedical engineering 2002, p365-368 